\title{
Lewis Base-Mediated Perovskite Crystallization as Revealed by In-Situ, Real-Time Optical Absorption Spectroscopy
}

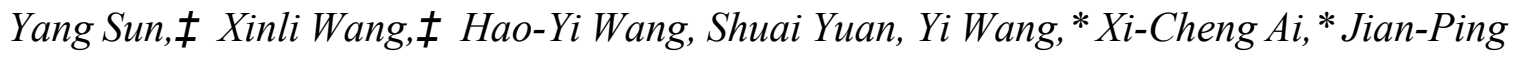

Zhang

Department of Chemistry, Renmin University of China, Beijing, 100872, China

AUTHOR INFORMATION

\section{Corresponding Authors:}

*Yi Wang, E-mail: ywang@ruc.edu.cn

*Xi-Cheng Ai, E-mail: xcai@ruc.edu.cn

\section{Experimental Sections}

\section{Perovskite film preparation.}

Substrate treatment. The quartz substrates $(1.5 \mathrm{~cm} \times 1.5 \mathrm{~cm} \times 0.2 \mathrm{~cm})$ were sequentially washed with aqueous detergent solution, deionized water, acetone, and ethanol for $30 \mathrm{~min}$, which was followed by a further oxygen plasma treatment for $10 \mathrm{~min}$.

Precursor preparation. $190.8 \mathrm{mg}$ of methylammonium iodide (MAI) and $553.2 \mathrm{mg}$ of 
$\mathrm{PbI}_{2}$ were dissolved in the mixed organic solvent comprising $700 \mu \mathrm{L}$ of $\mathrm{N}, \mathrm{N}-$ dimethylformamide (DMF), and $300 \mu \mathrm{L}$ of dimethylsulfoxide (DMSO). The as-obtained precursor solution was used to prepare pristine perovskite films, while for the urea-treated ones, 7.2 mg of urea was dissolved into the mixed solution additionally, and other procedures were kept the same.

Film fabrication (see Figure S2 for schematic illustration). $45 \mu \mathrm{L}$ of the precursor solution were spin-coated on the quartz substrates at $5000 \mathrm{rpm}$ for $35 \mathrm{~s}$. During the spincoating procedure, $300 \mu \mathrm{L}$ of chlorobenzene was dropped on the spinning film at the $22^{\text {nd }}$ s. After then, the as-obtained film was placed on the heating stage for $\sim 6 \mathrm{~min}$ at room temperature (corresponding to the "standing" period in Figure 2b). Subsequently, the heating stage was switched on with the desired annealing temperature. All the aforementioned procedures were carried out in a nitrogen-filled glove box.

\section{Basic characterizations.}

The morphologies of perovskite films were characterized by scanning electron microscopy (SEM, Hitachi SU8010) at an accelerating voltage of $4 \mathrm{kV}$. X-ray diffraction (XRD) analysis was conducted on a Shimadzu XRD-7000 diffractometer using $\mathrm{Cu} \mathrm{K} \alpha$ radiation in the $2 \theta$ ranging from $10^{\circ}$ to $45^{\circ}$ at a scan rate of $2^{\circ}$ per min. Time-correlated single-photon counting (TCSPC) data were acquired by using an Edinburgh FLS 980 
spectrometer, in which the sample was excited by a picosecond diode laser (EPL-475, excitation wavelength: $475 \mathrm{~nm}$, pulse width: $95 \mathrm{ps}$ ), and the detection wavelength was set as $770 \mathrm{~nm}$.

\section{In-situ, real-time absorption spectroscopy.}

The in-situ, real-time absorption spectroscopy measurement was performed in a vertical reflection mode, as illustrated in Figure 1 of the main text. In detail, the metal halide lamp (MHL, par38-E27, $70 \mathrm{~W}, 6000 \mathrm{~K}$ ), the spectrometer (AuaSpec-2048 × 16), and the customized sample stage placed on a heating stage (LEBO Science) were connected with a Y-shaped fiber. To maximize the spectra acquisition efficiency, a collimator was fixed on the top of the sample stage to assure the vertical alignment. A reflector (GMH006-AL, diameter: $6.3 \mathrm{~mm}$, thickness: $2 \mathrm{~mm}$ ) was located below the sample. The broadband incident light $(400 \mathrm{~nm}-850 \mathrm{~nm})$ from the MHL was directed to the sample stage through a collimator (diameter: $5 \mathrm{~mm}$, focal length: $10 \mathrm{~mm}$ ), which vertically passed through the sample and was then reflected by the reflector. The alignment was carefully optimized until the emergent light can be efficiently coupled into the Y-shaped fiber again, following which the wavelength-dependent intensity of the broadband light was recorded by the spectrometer with a sampling frequency of $4 \mathrm{~Hz}$. Baseline calibration has been conducted for all absorption spectra by measuring the spectral profile of the pure quartz substrate. 
(a)

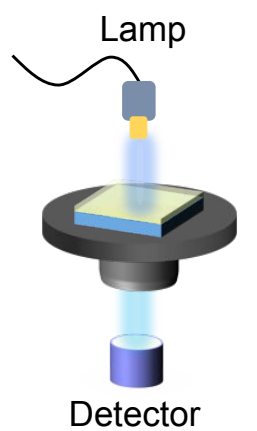

NOT available (b)
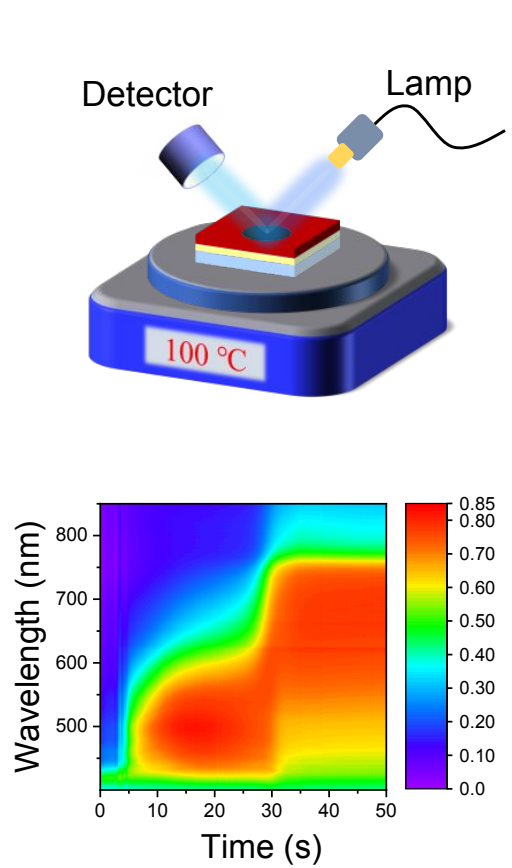

(c)
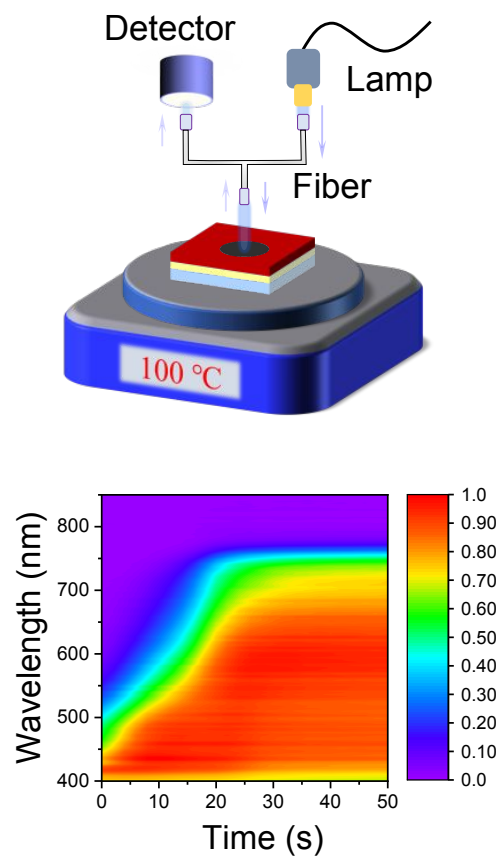

Figure S1. Comparison among the different operating modes of in-situ, real-time absorption spectroscopy: (a) transmission, (b) $45^{\circ}$-reflection, and (c) vertical reflection as adopted in this work. The upper panels show the optical geometries, and the corresponding lower panels show the representative spectroscopic data.

Figure S1 presents the optical alignments of the transmission, the $45^{\circ}$-reflection, and the vertical reflection-type in-situ, real-time absorption spectroscopy for the characterization of the optically active thin films during thermal annealing. The transmission mode (Figure S1a) will not be applicable, in case that the heating stage is not transparent enough. The $45^{\circ}$ reflection optical geometry has been widely used in literary works. ${ }^{\mathrm{S} 1}$, $\mathrm{S} 2$ However, the acquisition efficiency and especially the quality of the spectral 
data suffer from the interference from the scattering light from the film surface and the stray light. Consequently, the signal fluctuation, as seen from the diffusive fringes of the spectral map in Figure S1b (lower panel), may bring uncertainties in deriving the bandedge and the intragap state energies. In contrast to the $45^{\circ}$-reflection geometry, the newly devised vertical reflection one combined with the $\mathrm{Y}$-shaped fiber optics substantially improves the acquisition efficiency and, more importantly, effectively suppresses the interference from the scattering light from the sample surface and the stray light. These merits can be ascribed to the doubled optical pass of the white-light probe through a film, as well as to the conjugation effect of the collimator in sending out and collecting the probe light (Figure S1c, upper panel). The dramatic improvement of the data quality is obvious by comparing the distinctive spectral fringes in Figure S1c (lower panel) with the diffusive ones in Figure S1b (lower panel). 
(a)

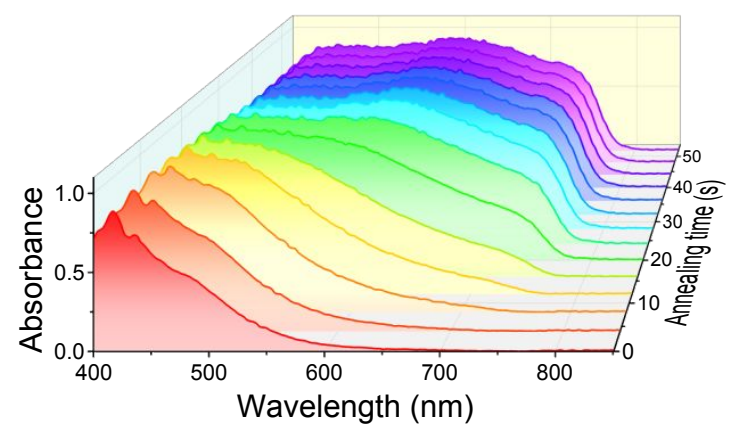

(b)

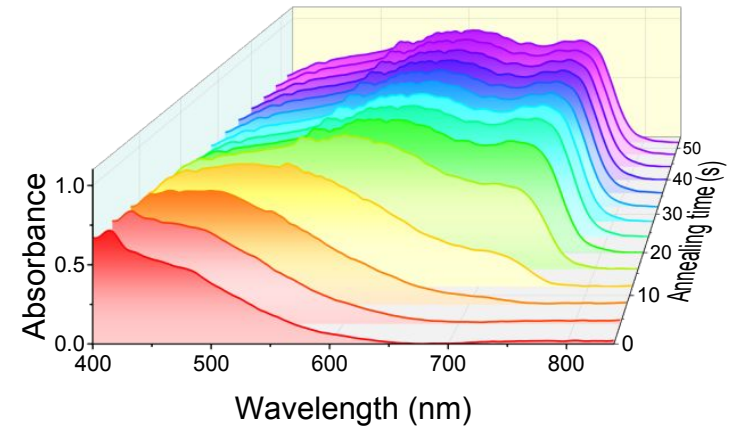

Figure S2. Representative absorption spectra of the pristine (a) and the urea-treated (b) perovskite films as a function of annealing time as extracted from Figure $2 \mathrm{a}$ and $2 \mathrm{~b}$ of the main text. 


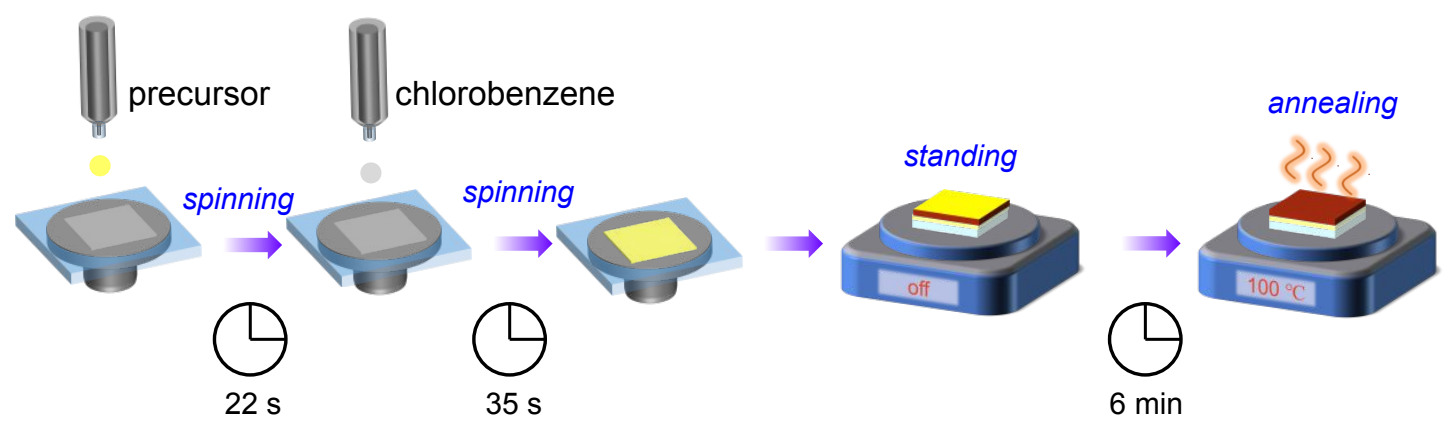

Figure S3. Schematic illustration of the film preparation procedures for in-situ, real-time absorption spectra acquisition.

The method of perovskite film preparation is schematically illustrated in Figure S3, and the relevant description is detailed in the Experimental Sections. It should be noted that the as-prepared films stand for $\sim 6$ min prior to being thermally annealed, which results in the two-stage in-situ, real-time absorption spectra as shown in Figure 2 of the main text. 

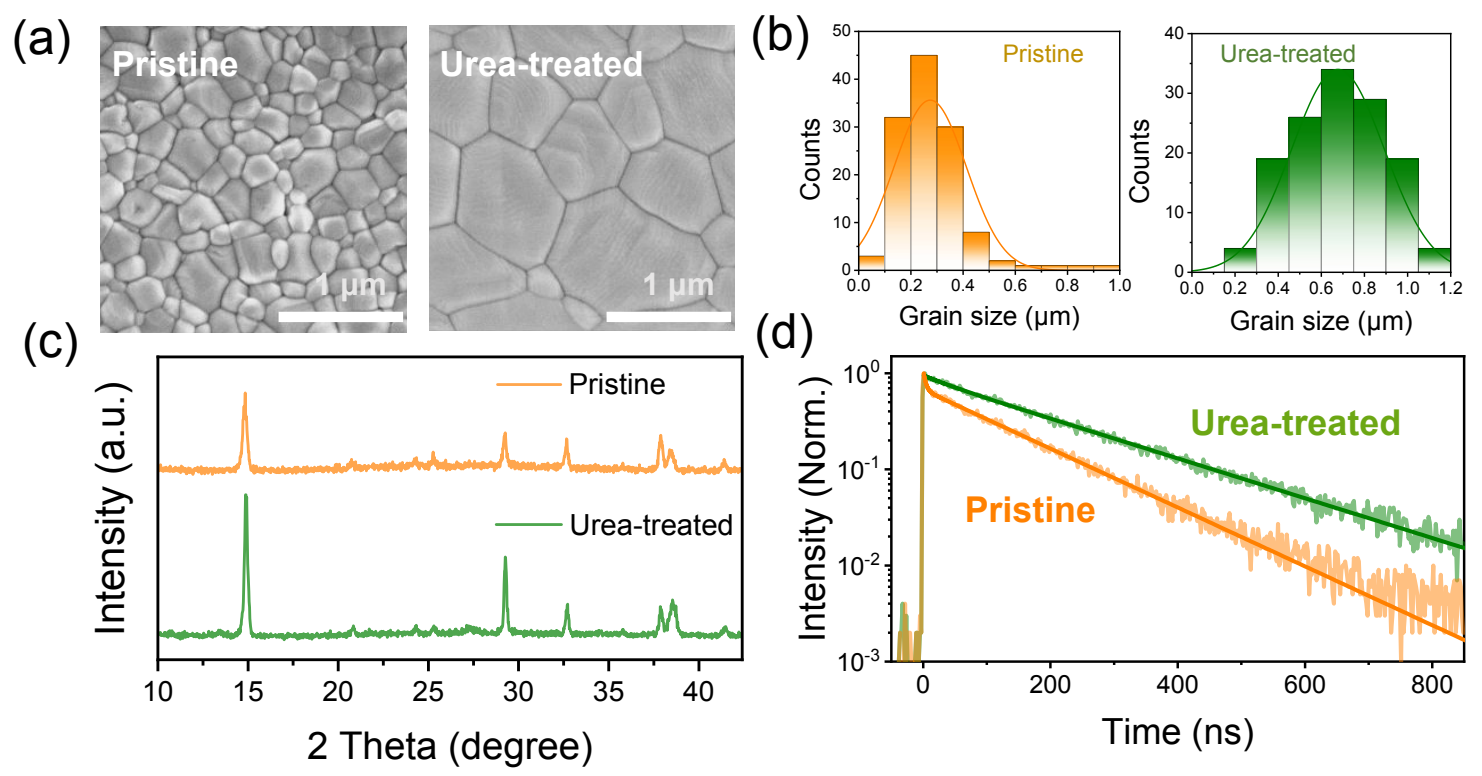

Figure S4. SEM images (a) and the corresponding size distribution histograms (b), XRD spectra (c), and time-resolved fluorescence traces (d) of the pristine and urea-treated perovskite films, respectively.

Scanning electronic microscopy (SEM) images of the pristine and urea-treated perovskite films are seen in Figure S4a. As expected, the grain size is dramatically enlarged by the urea treatment from $270 \mathrm{~nm}$ to $670 \mathrm{~nm}$ (Figure S4b). The X-ray diffraction (XRD) spectra (Figure S4c) exhibit an enhancement of the characteristic diffraction peaks, implying that the crystallinity of perovskite is ameliorated by the urea additive. The improved crystallinity usually links with reduced trap states, which in general acts as the nonradiative recombination centers of charge carriers, leading to prolonged fluorescence lifetime as revealed by the time-correlated single-photon counting (TCSPC) measurements (Figure S4d). These structural and spectroscopic characterization results collectively validate the positive effect of urea treatment on perovskite crystallization, which is in line 
with those reported ones and lends confidence to conduct in-depth studies on the underlying mechanism. ${ }^{\mathrm{S} 3-\mathrm{S} 5}$ 

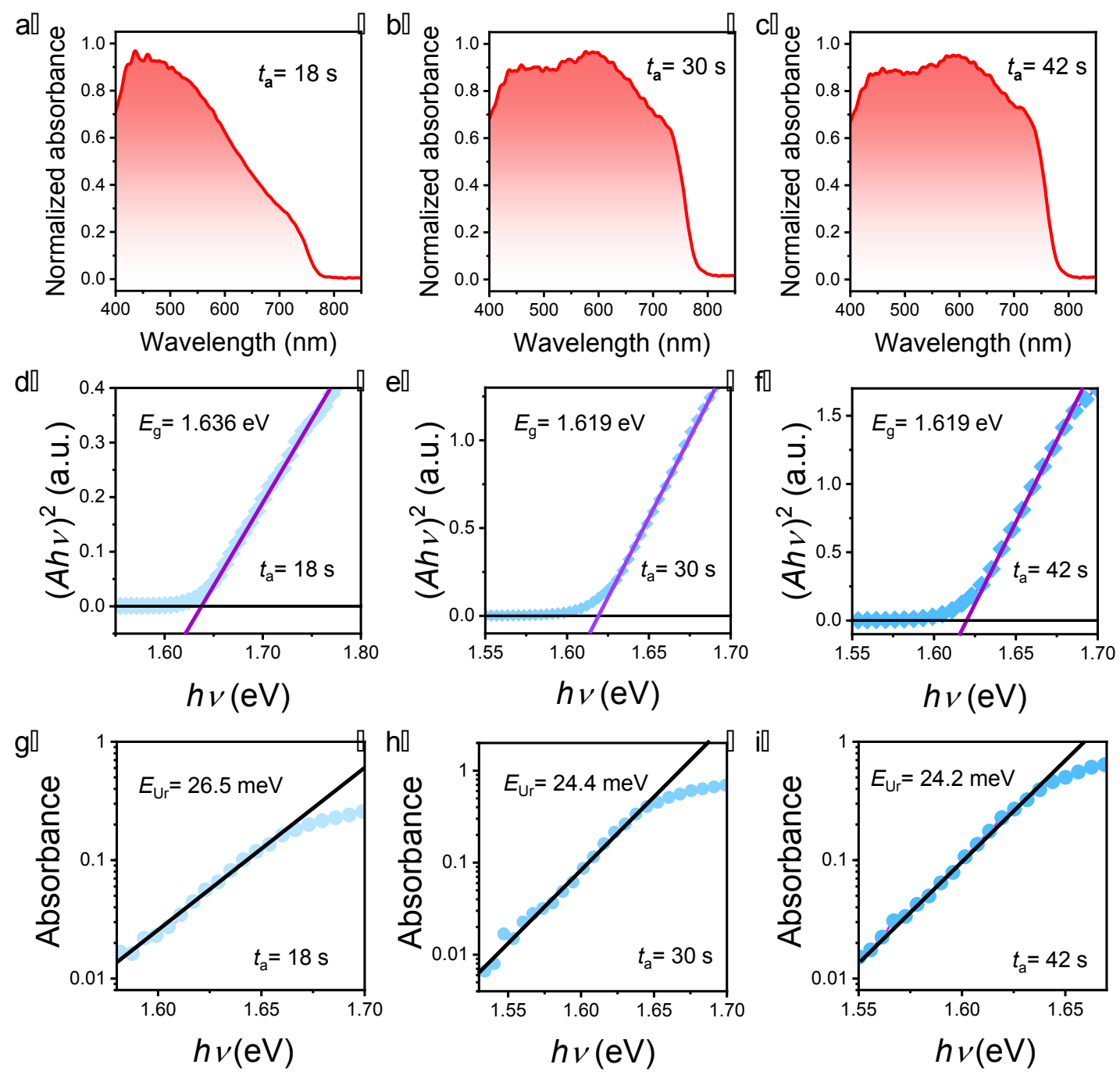

Figure S5. (a, b) Tauc plots and (c, d) Urbach plots derived from in-situ, real-time absorption spectra for the pristine perovskite films with different annealing time $t_{\mathrm{a}}$.

The optical bandgap $\left(E_{\mathrm{g}}\right)$ of perovskite films was calculated with the Tauc plot method.

In detail, the optical absorbance $(A)$ of semiconductors depends on the energy of the incident photon $(h v)$ in the form of $(A h v)^{1 / r}=B\left(h v-E_{\mathrm{g}}\right)$, where $B$ is a constant, and the factor $r$ equals $1 / 2$ in the case of direct bandgap semiconductors ${ }^{\mathrm{S} 6}$ such as $\mathrm{MAPbI}_{3}$ 
polycrystals. The Tauc plot works when $h v>E_{\mathrm{g}}$, therefore, by fitting the $h v$-dependent $(A h v)^{2}$ with a linear function, the value of $E_{\mathrm{g}}$ can be obtained from the intersection between the fitting function and the horizontal coordinate with a precision better than $10 \mathrm{meV}$. It has been widely reported that $\mathrm{MAPbI}_{3}$ films exhibit exponential decay of the density of states below $E_{\mathrm{g}}$, which is related to the electronic disorders, ${ }^{\mathrm{S}}, \mathrm{S} 8$ referred to as the Urbach tail. ${ }^{\mathrm{S} 9}$ Considering the proportional relationship between the density of the states and the absorbance, one can obtain the expression, $A=A_{0} \exp \left(h v / E_{\mathrm{Ur}}\right)$, where $E_{\mathrm{Ur}}$ is Urbach energy revealing the average energy level of the disorder states. Given the afore-mentioned equation, $E_{\mathrm{Ur}}$ can be straightforwardly derived from the linear fitting of $A-h v$ plot in a semi-logarithm scale. 
(a)
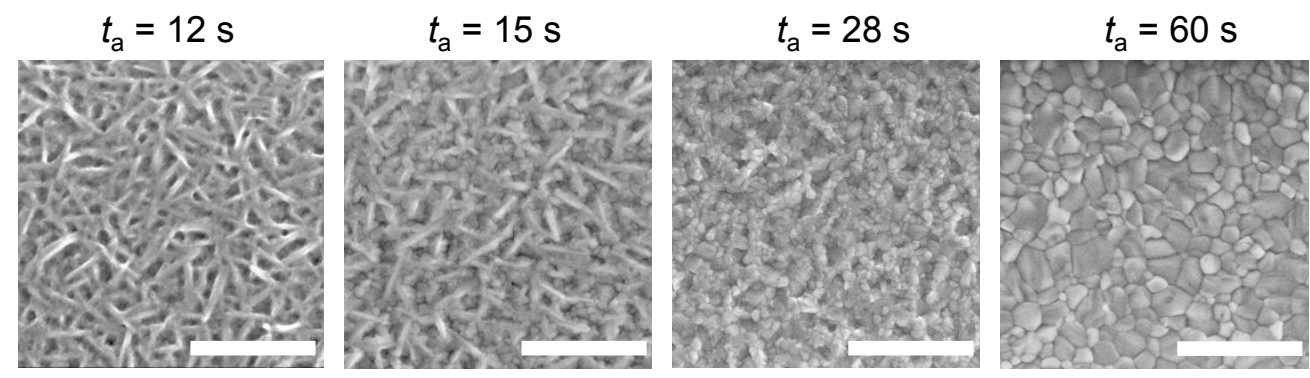

(b)
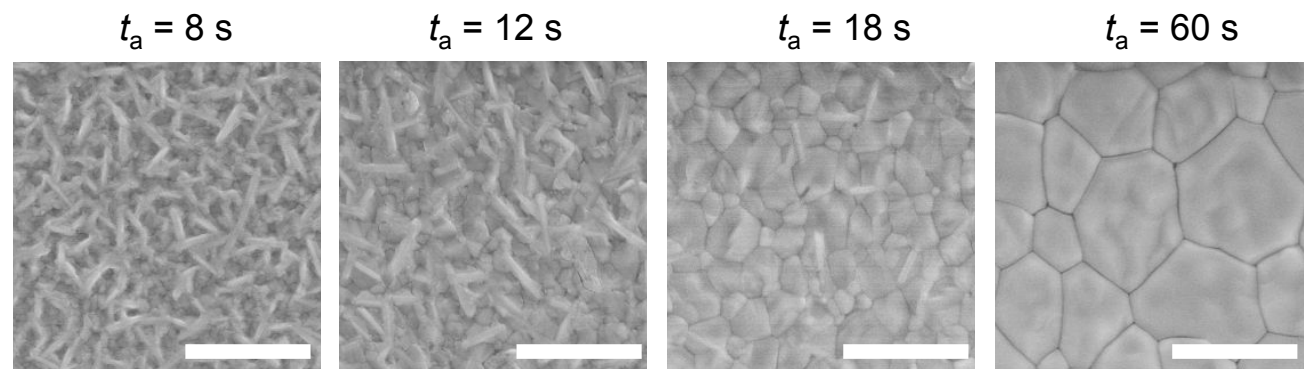

Figure S6. SEM top views of the pristine (a) and urea-treated (b) films annealed for different times (scale bar $=1 \mu \mathrm{m})$. 
(a)

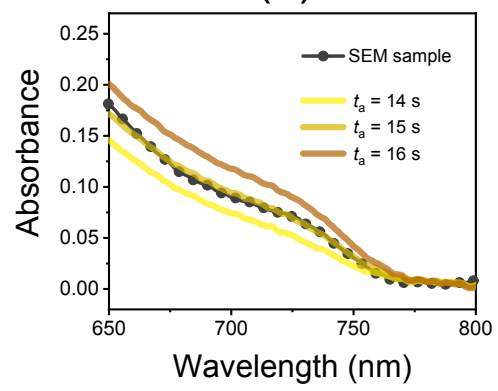

(b)

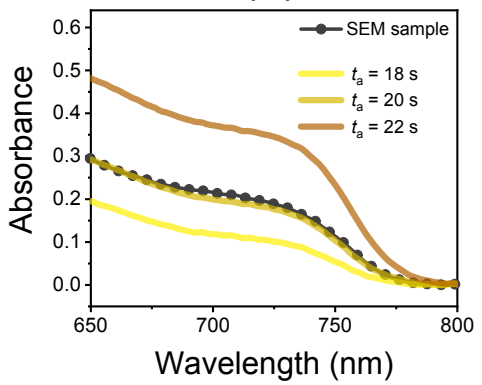

(c)

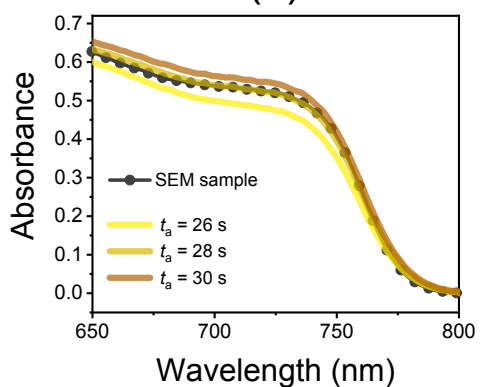

Figure S7. Time calibration for the SEM images. The solid curves are the in-situ, real-time absorption spectra with different $t_{\mathrm{a}}$, while the lines with circles are the absorption spectra of the films for SEM characterization with different recorded timings (see text below for details): $5 \mathrm{~s}(\mathrm{a}), 7 \mathrm{~s}(\mathrm{~b})$, and $12 \mathrm{~s} \mathrm{(c)}$.

To establish the accurate connection between the in-situ spectra and the SEM characterization results, the certain annealing time of the SEM samples should be calibrated first. The time directly read by the clock, referred to as the recorded timing, is not reliable, since the thermal annealing process is not immediately interrupted when the sample is removed from the heating stage. Fortunately, using the in-situ absorption spectroscopy, a reasonable time calibration method is readily established. The steady-state UV-vis absorption spectra of the SEM samples are plotted together with the in-situ absorption spectra at different $t_{\mathrm{a}}$. When the steady-state spectrum is consistent with an in-situ spectrum at a specific $t_{\mathrm{a}}$, the certain annealing time of the SEM sample is obtained. For example, the recorded timings of the SEM samples shown in Figure S7(a)-(c) are 5 s, 7 s, and 12 s, while after the time calibration procedures, the exact annealing times are obtained as $15 \mathrm{~s}, 20 \mathrm{~s}$, and $28 \mathrm{~s}$, respectively. 

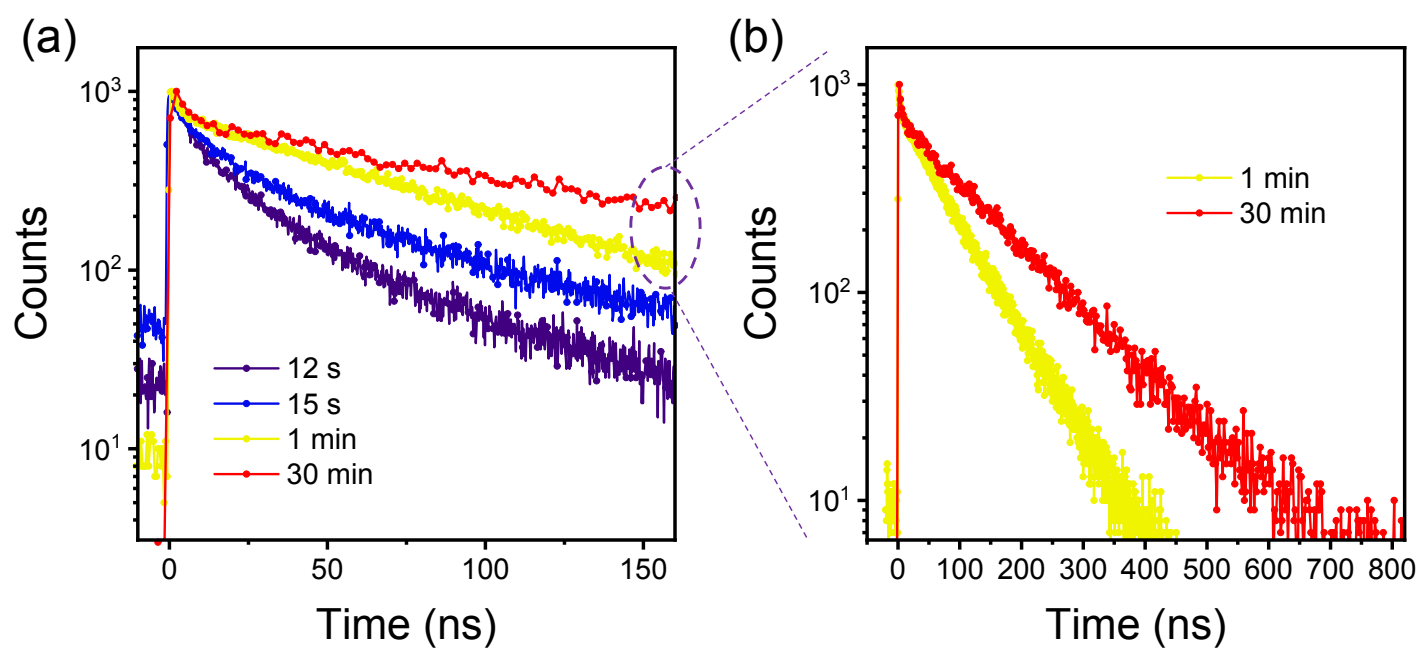

Figure S8. TCSPC traces (a) of the pristine perovskite films annealed for different times, and the zoomed-in plots (b) of the two curves corresponding to $t_{\mathrm{a}}=1 \mathrm{~min}$ and $30 \mathrm{~min}$, respectively. 
(a)

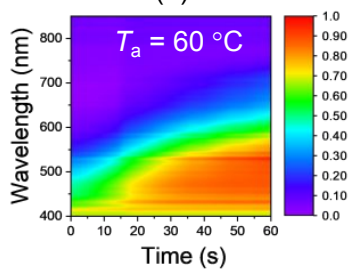

(e)

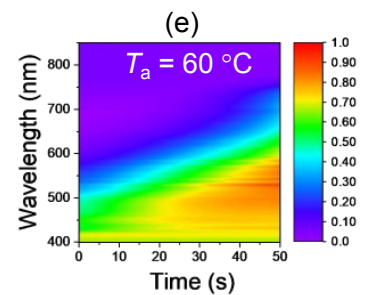

(b)
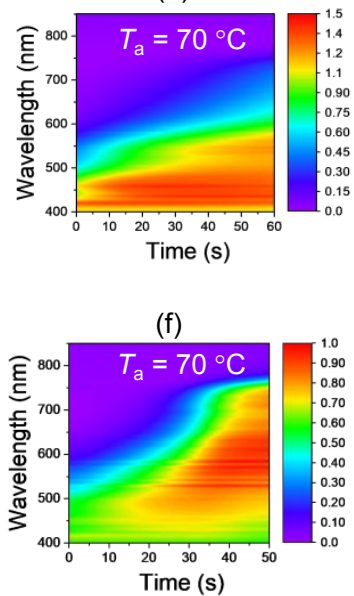

(c)

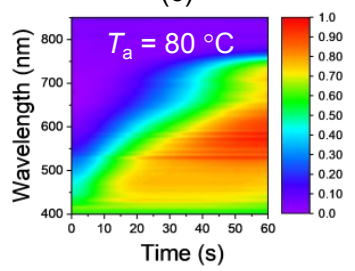

(g)

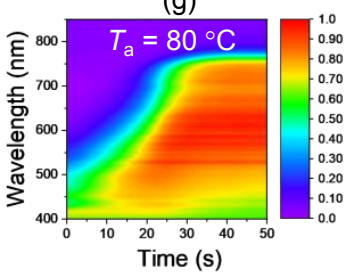

(d)

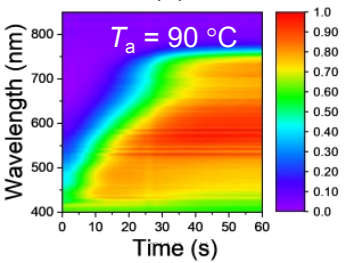

(h)

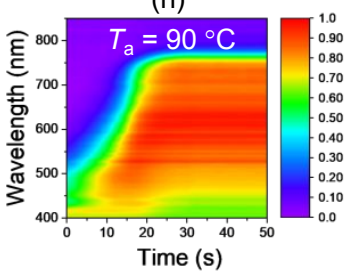

Figure S9. Representative in-situ, real-time absorption spectra of (a-d) the pristine and (e-h) the urea-treated films annealed at different temperatures. The data corresponding to $T_{\mathrm{a}}=$ $100^{\circ} \mathrm{C}$ have been shown in Figure 2 of the main text. 
(a)
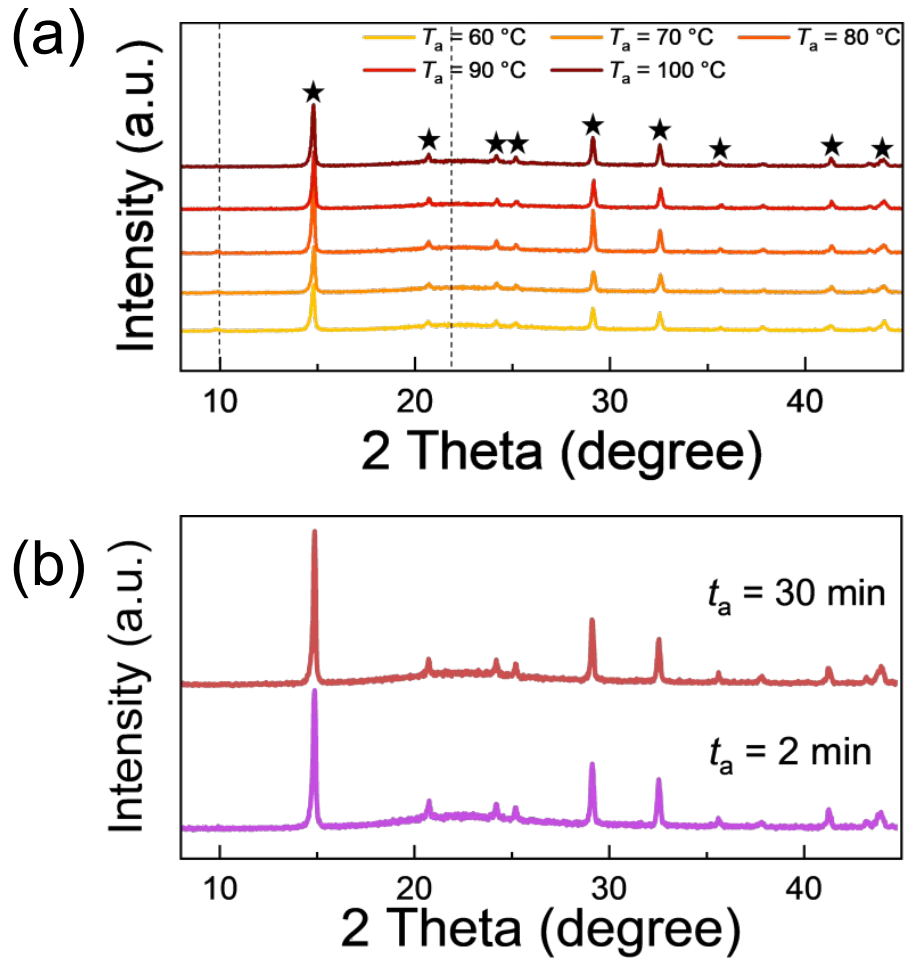

Figure S10. (a) XRD spectra of the pristine perovskite films annealed with different Ta. Stars and dashed lines, respectively, mark the characteristic diffraction peaks of the perovskite and the intermediates. (b) XRD spectra of the pristine perovskite films annealed at $100{ }^{\circ} \mathrm{C}$ for different $t_{\mathrm{a}}$.

Figure S10(a) shows the XRD spectra of the pristine perovskite films measured when the Abs@730 just got saturated. Since no significant intermediate signals were observed irrespective of $t_{\mathrm{a}}$, one may conclude that the crystallization reaction has been completed. This is further confirmed by Figure S10(b), showing that further elongation of $t_{\mathrm{a}}$ will no longer change the XRD patterns. 
(a)

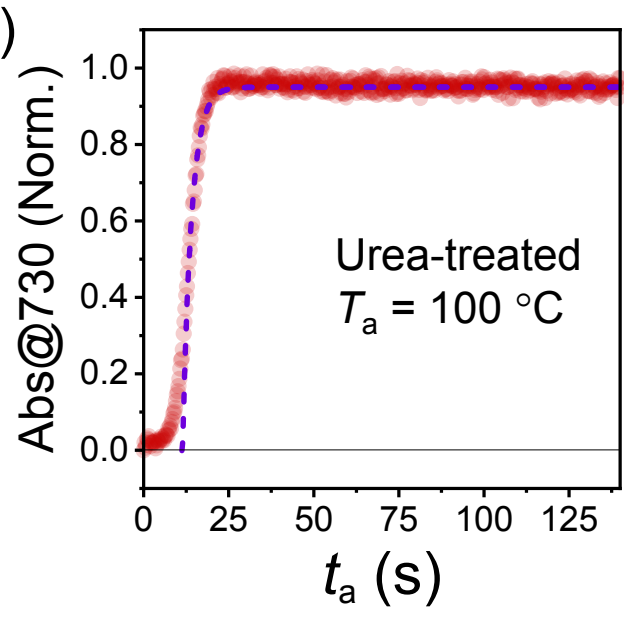

(b)

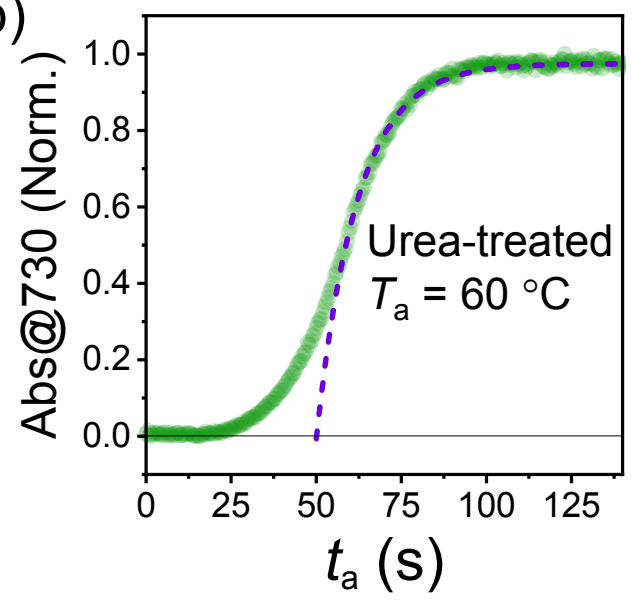

Figure S11. The time-dependent Abs@730 fitted with a single-exponential function within the framework of the first-order reaction kinetics. The circles are experimental data, and the dashed curves are fitting results.

Table S1. Crystallization rate constants $(k)$ of the pristine and urea-treated perovskite films as a function of annealing temperature $\left(T_{\mathrm{a}}\right)$.

\begin{tabular}{cccccc}
\hline$T_{\mathrm{a}}\left({ }^{\circ} \mathrm{C}\right)$ & 60 & 70 & 80 & 90 & 100 \\
\hline$k\left(10^{-2} \mathrm{~s}^{-1}\right)$ for the pristine film & 1.03 & 2.27 & 5.05 & 9.12 & 18.2 \\
$k\left(10^{-2} \mathrm{~s}^{-1}\right)$ for the urea-treated film & 8.78 & 14.4 & 21.2 & 32.3 & 52.1 \\
\hline
\end{tabular}

The function for fitting the Abs@730 - $t_{\mathrm{a}}$ plots can be written as below, which is as the first-order reaction model:

$$
A(t)=A_{0} \times\left[1-e^{-k\left(t_{a}-t_{0}\right)}\right]
$$

where $A(t)$ is the time-dependent absorbance at $730 \mathrm{~nm}, A_{0}$ is the maximum value of $A(t)$, 
and $k$ is the reaction rate constant. The parameter, $t_{0}$, is introduced by taking into account the heat transport effect; ${ }^{\mathrm{S} 1}$ that is, the real temperature of the heated sample cannot be enhanced to the set value immediately when the heating is switched on.

The $k$ values as a function of $T_{\mathrm{a}}$ obtained from the fitting results are summarized in Table S1 and visible in Figure 3c of the main text. The relationship between $k$ and the activation energy $\left(E_{\mathrm{a}}\right)$ is given by the Arrhenius equation, $k=A \exp \left(-E_{\mathrm{a}} / R T_{\mathrm{a}}\right)$, where $A$ is Arrhenius constant, and $R$ is the universal gas constant $\left(8.31 \mathrm{~J} \cdot \mathrm{mol}^{-1} \cdot \mathrm{K}^{-1}\right)$. After rewriting the Arrhenius equation as below, $\ln k=\ln A-E_{\mathrm{a}} / R T_{\mathrm{a}}$, one can obtain a linear dependence of $\ln k$ on $1 / T_{\mathrm{a}}$ with a slope of $-E_{\mathrm{a}} / R$. Therefore, by fitting the $\ln k-1 / T_{\mathrm{a}}$ plots with linear functions, the values of $E_{\mathrm{a}}$ can be derived from the slopes (Figure $3 \mathrm{c}$ of the main text) 


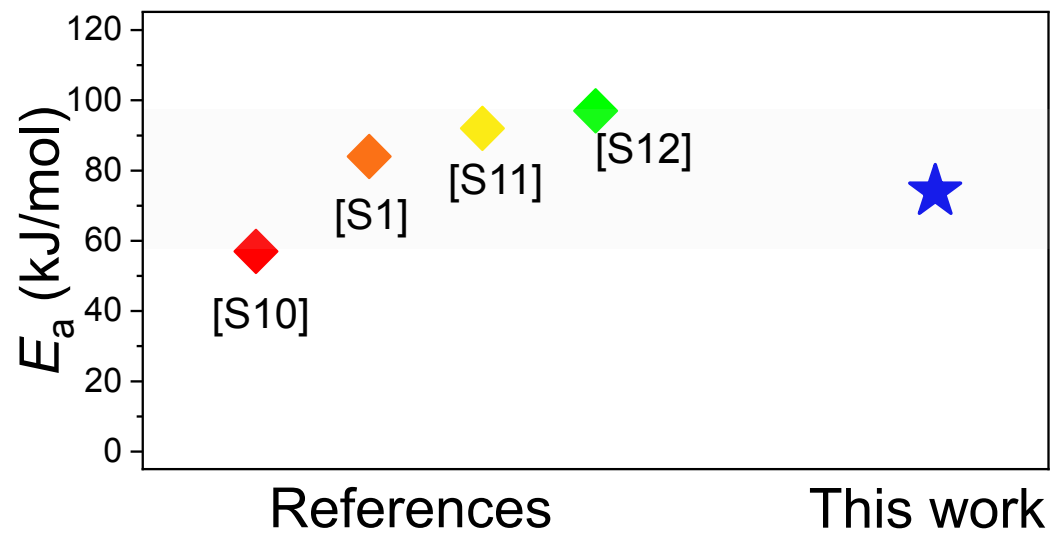

Figure S12. Comparison of the $E_{\mathrm{a}}$ value (blue star) obtained in the present work to those reported. ${ }^{\mathrm{S} 1, \mathrm{~S} 10-\mathrm{S} 12}$

Figure $\mathrm{S} 12$ summarizes the $E_{\mathrm{a}}$ of $\mathrm{MAPbI}_{3}$ crystallization. It is seen that the $E_{\mathrm{a}}$ value derived from our spectroscopic data $(\sim 73.7 \mathrm{~kJ} / \mathrm{mol})$ is in good accord with those reported $(55-100 \mathrm{~kJ} / \mathrm{mol}) . \mathrm{S} 1, \mathrm{~S} 10-\mathrm{S} 12$ 


\section{References:}

S1. Suchan, K.; Just, J.; Becker, P.; Unger, E. L.; Unold, T. Optical In Situ Monitoring during the Synthesis of Halide Perovskite Solar Cells Reveals Formation Kinetics and Evolution of Optoelectronic Properties. J. Mater. Chem. A 2020, 8, 10439-10449.

S2. Unger, E. L.; Bowring, A. R.; Tassone, C. J.; Pool, V. L.; Gold-Parker, A.; Cheacharoen, R.; Stone, K. H.; Hoke, E. T.; Toney, M. F.; McGehee, M. D. Chloride in Lead Chloride-Derived Organo-Metal Halides for Perovskite-Absorber Solar Cells. Chem. Mater. 2014, 26, 7158-7165.

S3. Lv, Y.; Zhang, H.; Wang, J.; Chen, L.; Bian, L.; An, Z.; Qian, Z.; Ren, G.; Wu, J.; Nuesch, F.; Huang, W. All-in-One Deposition to Synergistically Manipulate Perovskite Growth for High-Performance Solar Cell. Research 2020, 2020, 2763409.

S4. Han, L.; Cong, S.; Yang, H.; Lou, Y.; Wang, H.; Huang, J.; Zhu, J.; Wu, Y.; Chen, Q.; Zhang, B.; Zhang, L.; Zou, G. Environmental-Friendly Urea Additive Induced Large Perovskite Grains for High Performance Inverted Solar Cells. Sol. RRL 2018, 2, 1800054.

S5. Li, Y.; Li, L.; Yerramilli, A. S.; Chen, Y.; Fang, D.; Shen, Y.; Alford, T. L. Enhanced Power Conversion Efficiency and Preferential Orientation of the $\mathrm{MAPbI}_{3}$ Perovskite Solar Cells by Introduction of Urea as Additive. Org. Electron. 2019, 73, 130-136.

S6. Tauc, J. Optical Properties and Electronic Structure of Amorphous Ge and Si. Mat. Res. Bull. 1968, 3, $37-46$.

S7. De Wolf, S.; Holovsky, J.; Moon, S. J.; Loper, P.; Niesen, B.; Ledinsky, M.; Haug, F. J.; Yum, J. H.; Ballif, C. Organometallic Halide Perovskites: Sharp Optical Absorption Edge and Its Relation to Photovoltaic Performance. J. Phys. Chem. Lett. 2014, 5, 1035-1039.

S8. Sadhanala, A.; Deschler, F.; Thomas, T. H.; Dutton, S. E.; Goedel, K. C.; Hanusch, F. C.; Lai, M. L.; Steiner, U.; Bein, T.; Docampo, P.; Cahen, D.; Friend, R. H. Preparation of Single-Phase Films of $\mathrm{CH}_{3} \mathrm{NH}_{3} \mathrm{~Pb}\left(\mathrm{I}_{1-\mathrm{x}} \mathrm{Br}_{\mathrm{x}}\right)_{3}$ with Sharp Optical Band Edges. J. Phys. Chem. Lett. 2014, 5, 2501-2505.

S9. Lax, M. Generalized Mobility Theory. Phys. Rev. 1958, 109, 1921-1926.

S10. Song, J.; Hu, Q.; Zhang, Q.; Xiong, S.; Zhao, Z.; Ali, J.; Zou, Y.; Feng, W.; Yang, Z.; Bao, Q.; Zhang, Y.; Russell, T. P.; Liu, F. Manipulating the Crystallization Kinetics by Additive Engineering toward High-Efficient Photovoltaic Performance. Adv. Funct. Mater. 2021, 31, 2009103.

S11. Khlyabich, P. P.; Loo, Y.-L. Crystalline Intermediates and Their Transformation Kinetics during the Formation of Methylammonium Lead Halide Perovskite Thin Films. Chem. Mater. 2016, 28, 9041-9048.

S12. Moore, D. T.; Sai, H.; Tan, K. W.; Smilgies, D.-M.; Zhang, W.; Snaith, H. J.; Wiesner, U.; Estroff, L. A. Crystallization Kinetics of Organic-Inorganic Trihalide Perovskites and the Role of the Lead Anion in Crystal Growth. J. Am. Chem. Soc. 2015, 137, 2350-2358. 